\section{Prevalência de transtornos mentais comuns em mulheres e sua relação com as características sociodemográficas e o trabalho doméstico}

\author{
Prevalence of psychological disorders \\ among women according to socio \\ demographic and housework characteristics
}

Tânia Maria de Araújo 1

Paloma de Sousa Pinho 2

Maura Maria Guimarães de Almeida 3
1-3 Mestrado em Saúde Coletiva. Módulo VI. Núcleo de Epidemiologia. Departamento de Saúde. Universidade Estadual de Feira de Santana. Centro de Pós-Graduação em Saúde Coletiva. Km 3, BR 116. Campus Universitário. Feira de Santana, BA, Brasil. CEP: $44.031-460$

\begin{abstract}
Objectives: to estimate minor mental disorders prevalence $(M D P)$ in women and to describe related factors with emphasis on domestic work.

Methods: cross sectional study with women in Feira de Santana, Bahia. Two thousand and fifty five women (2055) aged $\geq 15$ years old were interviewed. Social and demographic characteristics and domestic work aspects were assessed. Mental health was assessed through Reporting Questionnaire (SRQ-20). Minor mental disorders prevalence and association with other factors was calculated through prevalence ratio.

Results: global MDP was of 39.4\% (IC95\%: 37.3$41.6 \%)$. Women with high domestic workload had high MDP (48.1\%) compared with women with low domestic workloads (22.5\%). Hired domestic help in accomplishing domestic work was associated to low MDP (28.0\%) and high prevalence in women who did not receive any help $(47.1 \%)$ or had only the help of one man (46.9\%). Other features were associated to MDP: being negro or mulatto, divorced/separated/ widow, low education level, or low income, having children, being the family head and not dedicating weekly time to leisure.

Conclusions; aspects related to domestic work should be considered and incorporated to mental health assessment in women.
\end{abstract}

Key words Work, Mental disorders, Mental health, Women

\section{Resumo}

Objetivos: estimar a prevalência de transtornos mentais comuns (TMC) em mulheres e descrever fatores associados à sua ocorrência, com ênfase no trabalho doméstico.

Métodos: estudo de corte transversal com mulheres de Feira de Santana, Bahia. Foram entrevistadas 2055 mulheres, com idade $\geq 15$ anos. Avaliaram-se características sociodemográficas e aspectos do trabalho doméstico. A saúde mental foi avaliada pelo Self Reporting Questionnaire (SRQ-20). Calculou-se a prevalência de transtornos mentais comuns, sua associação com alguns fatores, mediante o cálculo da razão de prevalência.

Resultados: a prevalência global de TMC foi 39,4\% (IC95\%: 37,3-41,6\%). Mulheres com alta sobrecarga doméstica apresentaram prevalência de TMC mais elevada $(48,1 \%)$ do que mulheres com baixa sobrecarga (22,5\%). Ajuda doméstica remunerada na realização das tarefas associou-se à baixa prevalência de TMC (28,0\%); e elevadas prevalências em mulheres que não recebiam ajuda $(47,1 \%)$ ou contavam apenas com o auxílio de um homem (46,9\%). Outras características estavam associadas à ocorrência de TMC: ser negra ou parda, divorciada/desquitada/viúva, baixo nível de escolaridade, ou de renda, ter filhos, ser chefe de família e não dedicar tempo semanal ao lazer.

Conclusões: aspectos referentes ao trabalho doméstico devem ser considerados e incorporados à avaliação da saúde mental das mulheres.

Palavras-chave Trabalho, Transtornos mentais, Saúde mental, Mulheres 


\section{Introdução}

A literatura tem registrado, nos últimos anos, uma preocupação crescente com o diagnóstico e a avaliação da saúde mental das populações. Dentre os efeitos avaliados, destacam-se os transtornos mentais comuns (TMC), expressão criada por Goldberg e Huxley ${ }^{1}$ para caracterizar sintomas como insônia, fadiga, irritabilidade, esquecimento, dificuldade de concentração e queixas somáticas. Em termos gerais, transtornos mentais comuns designam situações de sofrimento mental. ${ }^{2}$

Populações ocidentais apresentam prevalências de transtornos mentais não psicóticos que variam de $7 \%$ a $26 \%$, sendo mais elevadas no sexo feminino $(20,0 \%)$ do que no masculino $(12,5 \%) .3$ Os quadros depressivos correspondem ao terceiro problema de saúde entre mulheres residentes em países desenvolvidos e ao quinto entre as mulheres de países em desenvolvimento. Além dos sintomas depressivos, entre os transtornos mentais que predominam entre as mulheres, estão aqueles relacionados aos fatores psicossociais e ambientais, como transtornos de ansiedade, transtornos de ajustamento, insônia, estresse, transtorno da alimentação e anorexia nervosa. 4

Além das repercussões negativas sobre a saúde implicando adoecimento psíquico, aspectos associados à saúde mental crescem em relevância quando avaliadas suas repercussões em outras esferas da vida. Tem sido observado, por exemplo, que indivíduos com transtornos mentais comuns apresentam taxas de mortalidade mais elevadas 5 e que indivíduos com TMC apresentam, em média, duas vezes mais queixas de saúde física do que aqueles sem TMC. 6

Em estudos conduzidos em comunidades, mulheres têm apresentado consideravelmente mais sintomas de angústia psicológica e desordens depressivas do que homens. ${ }^{7}$ Dada a magnitude de adoecimento psíquico entre as mulheres, a avaliação de fatores associados à ocorrência de TMC é, portanto, uma tarefa de grande relevância, podendo fornecer base empírica para intervenções.

Ludermir e Melo Filho ${ }^{2}$ destacam que a epidemiologia psiquiátrica tem verificado associação entre TMC e variáveis relativas às condições de vida e à estrutura ocupacional. Nos últimos anos, vem crescendo o número de estudos que incluíram a avaliação de aspectos relacionados ao trabalho profissional, decorrente da inserção da mulher no mercado de trabalho, como fator associado, determinante ou contribuinte para a ocorrência de TMC observada.
Segundo Araújo, ${ }^{8}$ entre os estudos sobre o papel do trabalho na vida das mulheres destacam-se aqueles que enfocam os seus papéis sociais e, que geralmente comparam padrões de ocorrência de agravos à saúde mental entre mulheres inseridas no mercado de trabalho e donas-de-casa. Em estudos compa-rando mulheres com trabalho remunerado e donas-de-casa, foi observado que fatores distintos produziam adoecimento psíquico e recompensa entre esses dois tipos de ocupação. Donas-de-casa e trabalhadoras experimentaram, em média, níveis similares de sintomas depressivos, ainda que os fatores associados à ocorrência de adoecimento psíquico tenham sido distintos para cada grupo. ${ }^{9}$ Dentre os aspectos referentes ao trabalho doméstico associados aos sintomas depressivos, ansiosos ou psicossomáticos destacam-se a rotinização e as interrupções constantes das atividades realizadas.

Ao contrário do que ocorre com os homens, a inserção feminina no mercado produtivo é limitada por suas responsabilidades domésticas e familiares, tendo o emprego que ser adaptado à estas funções. 10

Apesar da peculiaridade do trabalho profissional das mulheres, limitado ou acrescido por responsabilidades domésticas, aspectos relacionados ao trabalho doméstico ainda permanecem pouco estudados. Uma das razões para essa invisibilidade social decorre da própria percepção deste tipo de atividade como não-trabalho, invisível na produção social, uma vez que não produz valor. ${ }^{11} \mathrm{~A}$ ausência de reconhecimento pelo trabalho realizado emerge como conseqüência dessa invisibilidade e, não raro, aparece como produtor de intenso sofrimento psíquico.

O sofrimento associado ao trabalho doméstico decorre de tensões geradas pelas suas características de monotonia, repetitividade, desvalorização e pelas demandas dos papéis sociais aos quais a mulher deve atender. ${ }^{12}$ A vivência cotidiana dessas tensões, acumuladas ao longo do tempo, pode, como apontam vários estudos, 8,9 cristalizar diferentes formas de adoecimento psíquico.

Considerando que o adoecimento psíquico tem sido mais prevalente entre as mulheres e que poucos estudos têm avaliado os fatores associados à sua ocorrência na realidade brasileira, este estudo objetivou: a) descrever características sociodemográficas e do trabalho doméstico de mulheres de Feira de Santana; b) estimar a prevalência de transtornos mentais comuns nessas mulheres e c) descrever fatores associados à prevalência de transtornos mentais comuns, com ênfase nos aspectos relacionados ao trabalho doméstico. 


\section{Métodos}

Realizou-se estudo epidemiológico de corte transversal, com finalidade descritiva, exploratória. Fo estudada uma amostra representativa da população com idade de 15 anos ou mais, residente na zona Urbana de Feira de Santana, Bahia, Brasil.

Assumindo-se prevalência estimada de transtornos mentais comuns entre mulheres de $25 \%, 13$ erro amostral de 3\%, com $95 \%$ de confiança, calculou-se uma amostra de 800 indivíduos, seguindo-se os procedimentos recomendados por Lwanga e Lemeshow. ${ }^{14}$ Considerando-se o efeito do desenho do estudo, duplicou-se o tamanho da amostra para 1600 pessoas. Admitindo-se recusas e perdas em torno de $20 \%$, definiu-se um tamanho amostral de 1920 .

A seleção das áreas foi feita por amostragem estratificada por subdistrito, adotando-se procedimento aleatório, a partir de dados censitários do Instituto Brasileiro de Geografia e Estatística (IBGE). 15 Sortearam-se, inicialmente, os setores censitários em cada subdistrito da zona urbana e, em seguida, foram sorteadas as ruas, em cada setor censitário selecionado. Todos os domicílios das ruas sorteadas foram visitados e todas as mulheres com 15 anos ou mais de idade foram consideradas elegíveis para o estudo e entrevistadas por pesquisadores previamente treinados. Para reduzir o percentual de perdas, adotou-se o procedimento de realizar até três visitas por domicílio. Foram visitados 1479 domicílios e entrevistadas 2055 mulheres.

Para a coleta de dados utilizaram-se: a) ficha domiciliar (com dados gerais sobre o domicílio e seus residentes) e b) questionário individual (com informações sociodemográficas, acerca do trabalho profissional, do trabalho doméstico, atividades de lazer e saúde mental). Os instrumentos de pesquisa foram previamente testados em um estudo piloto. 16

Idade, situação conjugal, escolaridade, cor da pele, renda mensal, chefia da família e presença de filhos foram as características sociodemográficas investigadas neste estudo. "Trabalho doméstico" foi avaliado segundo os critérios propostos por Aquino17 e incluiu: tipos de atividades domésticas realizadas, grau de responsabilidade assumida na execução das tarefas domésticas, frequiência semanal em que se realizavam as tarefas e apoio recebido na sua realização.

Os transtornos mentais comuns (TMC) foram avaliados utilizando-se o Self-Reporting Questionnaire (SRQ-20). O SRQ-20 é um instrumento de triagem de morbidade psíquica neurótica, desenhado pela Organização Mundial da Saúde (OMS) para uso em populações de países em desenvolvimento. ${ }^{18}$ Como é um instrumento para detecção de sintomas, é adequado para estudos populacionais 8 com a finalidade de detectar presença de sintomas em geral - caracterização de grau de suspeição de transtorno psíquico. No Brasil, o SRQ-20 tem sido utilizado para estudos em serviços de atenção primária à saúde 18 e em grupos ocupacionais específicos. 8,19 Estudos de validação revelaram desempenho satisfatório do SRQ-20 na realidade brasileira. 20 No presente estudo, definiu-se o ponto de corte para classificação de suspeitos de TMC em sete ou mais respostas positivas, procedimento adotado em outros estudos. $6,8,19$

Taxas de prevalência, razões de prevalência de TMC e seus respectivos intervalos de $95 \%$ de confiança foram calculados para avaliar, exploratoriamente, a associação entre os fatores estudados e prevalência de TMC. O teste qui-quadrado foi utilizado para avaliar associação entre TMC e variáveis relacionadas ao trabalho doméstico.

A pesquisa foi submetida ao Comitê de Ética em Pesquisa do Hospital São Rafael, em Salvador, Bahia, tendo sido aprovada. Todos os indivíduos que aceitaram participar da pesquisa assinaram termo de consentimento segundo as recomendações da Comissão Nacional de Ética em Pesquisa (CONEP) e da Resolução 196/96 sobre Ética em Pesquisa com Seres Humanos.

\section{Resultados}

Entre as mulheres entrevistadas $(\mathrm{N}=2055)$ predominaram as casadas ou em união estável $(46,6 \%)$, com menos de 41 anos $(63,1 \%)$, de cor parda $(56,0 \%)$, com baixo nível de escolaridade $(13,1 \%$ não freqüentaram a escola e $44,1 \%$ tinham apenas o ensino fundamental) (Tabela 1 ). $\mathrm{O}$ rendimento próprio mensal foi muito baixo $(18,6 \%$ contavam com menos de meio salário mínimo e 39,3\% recebiam até um salário mínimo). Cerca de $1 / 3$ das mulheres não tinha filhos; dentre as que referiram ter filhos, predominaram aquelas com um a dois filhos (30,9\%); 7,1\% relataram ter mais de oito filhos.

Considerando a ocupação da entrevistada no momento da pesquisa, 652 mulheres $(31,7 \%)$ estavam trabalhando, 233 (11,3\%) estavam desempregadas, $583(28,4 \%)$ sempre foram donas-de-casa, $176(8,6 \%)$ eram aposentadas, $32(1,6 \%)$ encontravam-se afastadas do trabalho profissional por problema de saúde e $296(14,4 \%)$ eram apenas estudantes. 
Tabela 1

Características sociodemográficas das mulheres de Feira de Santana, Bahia, 2002.

\begin{tabular}{|c|c|c|}
\hline Característica & $\mathbf{N}$ & $\%$ \\
\hline \multicolumn{3}{|l|}{ Faixa etária (anos) ( $\mathrm{N}=2055)$} \\
\hline Até 20 & 434 & 21,1 \\
\hline $21-30$ & 470 & 22,9 \\
\hline $31-40$ & 392 & 19,1 \\
\hline $41-50$ & 274 & 13,3 \\
\hline$>50$ & 485 & 23,6 \\
\hline \multicolumn{3}{|l|}{ Escolaridade $(\mathrm{N}=2038)$} \\
\hline Nunca foi à escola & 267 & 13,1 \\
\hline Ensino fundamental (I Grau) & 898 & 44,1 \\
\hline Ensino médio (II Grau) & 797 & 39,1 \\
\hline Superior & 76 & 3,7 \\
\hline \multicolumn{3}{|l|}{ Situação conjugal $(N=2047)$} \\
\hline Solteira & 737 & 36,0 \\
\hline Casada/união estável & 954 & 46,6 \\
\hline Divorciada/desquitada & 164 & 8,0 \\
\hline Viúva & 192 & 9,4 \\
\hline \multicolumn{3}{|l|}{ Rendimento próprio mensal $(\mathrm{N}=822)^{*}$} \\
\hline Até $\mathrm{R} \$ 100,00$ (até $1 / 2$ salário mínimo) & 153 & 18,6 \\
\hline $\mathrm{R} \$ 101,00-200,00$ (até $1 \mathrm{SM}$ ) & 323 & 39,3 \\
\hline $\mathrm{R} \$ 201,00-400,00$ (até $2 \mathrm{SM}$ ) & 200 & 24,3 \\
\hline$>\mathrm{R} \$ 401,00$ (mais de $2 \mathrm{SM}$ ) & 146 & 17,8 \\
\hline \multicolumn{3}{|l|}{ Cor da Pele $(N=2035)$} \\
\hline Branca & 435 & 21,4 \\
\hline Parda & 1.140 & 56,0 \\
\hline Preta & 352 & 17,3 \\
\hline Amarela/ Indígena & 24 & 1,2 \\
\hline Não sabe & 84 & 4,1 \\
\hline \multicolumn{3}{|l|}{ Número de filhos $(\mathrm{N}=2055)$} \\
\hline Não tinha filhos & 659 & 32,1 \\
\hline 1 a 2 filhos & 636 & 30,9 \\
\hline 3 a 4 filhos & 393 & 19,1 \\
\hline 5 a 7 filhos & 222 & 10,8 \\
\hline Mais de 8 filhos & 145 & 7,1 \\
\hline
\end{tabular}

* Apenas $822(40 \%)$ das mulheres referiram ter algum tipo de rendimento próprio; SM = salário mínimo (valor vigente na época: $R \$ 200,00$.).

Dentre as mulheres entrevistadas, $13,7 \%$ era chefe de família. As tarefas domésticas eram realizadas todos os dias da semana por $83,8 \%$, enquanto apenas $5,3 \%$ não faziam qualquer tipo de trabalho doméstico; $25,4 \%$ das mulheres não recebiam qualquer tipo de ajuda na realização das tarefas domésticas, e quando havia o apoio, em 51,8\% dos casos, esse era disponibilizado por outras mulheres ou por empregada (12,2\%) (Tabela 2). A sobrecarga doméstica alta, indicada pelas atividades de cozinhar, lavar, passar e limpar, foi referida por $26,9 \%$ das mulheres.
Em todos os tipos de atividade doméstica investigadas, a proporção de mulheres que executava tarefas domésticas foi sempre superior à proporção daquelas que não executava nenhum tipo de atividade. Tarefas como cuidar das crianças e cozinhar destacaram-se como as mais freqüentemente referidas; enquanto para tarefas como lavar e passar observou-se decréscimo na freqüência das mulheres que referiram ser a principal responsável pela execução dessas tarefas (Tabela 3 ).

Um percentual expressivo não mantinha ativi- 
dades regulares de lazer $(45,1 \%)$. O percentual de mulheres que dedicava tempo semanal ao lazer diminuía com o aumento do número de filhos: foi mais elevado entre as mulheres sem filhos (corres- pondia a $72,8 \%$ das mulheres) e menor entre aquelas com cinco a sete filhos (apenas $35,6 \%$ referiram ter algum tipo de atividade de lazer) e com oito filhos ou mais $(40,7 \%)$ (Tabela 4$)$.

Tabela 2

Distribuição das mulheres segundo características do trabalho doméstico.

\begin{tabular}{|c|c|c|}
\hline Característica & $\mathbf{N}$ & $\%$ \\
\hline \multicolumn{3}{|l|}{ Chefia da família } \\
\hline A própria & 281 & 13,7 \\
\hline Outro chefe & 1774 & 86,3 \\
\hline \multicolumn{3}{|l|}{ Ajuda nas tarefas domésticas } \\
\hline Nenhuma & 497 & 25,4 \\
\hline Outra mulher & 1011 & 51,8 \\
\hline Empregada & 239 & 12,2 \\
\hline Marido/outro homem & 147 & 7,5 \\
\hline Outra alternativa & 59 & 3,0 \\
\hline \multicolumn{3}{|c|}{ Dias da semana que realiza tarefas } \\
\hline \multicolumn{3}{|c|}{ domésticas } \\
\hline Todos os dias & 1664 & 83,8 \\
\hline Segunda a sexta & 43 & 2,2 \\
\hline Apenas finais de semana & 173 & 8,7 \\
\hline Não faz & 106 & 5,3 \\
\hline \multicolumn{3}{|l|}{ Sobrecarga doméstica } \\
\hline Baixa & 720 & 35,2 \\
\hline Média & 775 & 37,9 \\
\hline Alta & 550 & 26,9 \\
\hline
\end{tabular}

Tabela 3

Percentual das atividades domésticas realizadas segundo o grau de responsabilidade das mulheres.

\begin{tabular}{lccc}
\hline Atividade & Não faz & Faz a menor & $\begin{array}{c}\text { Faz a maior } \\
\text { parte / divide }\end{array}$ \\
\hline parte / inteiramente \\
\hline Cuidar de crianças* & $\%$ & $\%$ & $\%$ \\
Cozinhar & 19,6 & 34,2 & 46,2 \\
Limpeza da casa & 17,0 & 36,3 & 46,7 \\
Lavar roupas & 10,7 & 45,3 & 44,0 \\
Passar roupas & 19,4 & 38,3 & 42,3 \\
\hline
\end{tabular}

* Incluiu apenas mulheres com crianças pequenas (até 12 anos) 
Tabela 4

Distribuição das mulheres segundo atividades de lazer e número de filhos.

\begin{tabular}{lrrrrrr}
\hline Número de filhos & Atividades de lazer & & & \\
\hline & \multicolumn{2}{c}{ Sim } & & Não & Total \\
\hline & $\mathrm{n}$ & $\%$ & $\mathrm{n}$ & $\%$ & \\
\hline Sem filhos & 480 & 72,8 & 179 & 27,2 & 659 \\
1 a 2 filhos & 326 & 51,3 & 309 & 48,7 & 635 \\
3 a 4 filhos & 183 & 46,6 & 210 & 53,4 & 393 \\
5 a 7 filhos & 79 & 35,6 & 143 & 64,4 & 222 \\
8 ou mais filhos & 59 & 40,7 & 86 & 59,3 & 145 \\
Total & 1127 & 54,9 & 927 & 45,1 & 2.054 \\
\hline
\end{tabular}

Tabela 5

Prevalência (\%) dos grupos de sintomas psíquicos avaliados pelo SelfReporting Questionnaire (SRQ-20).

\begin{tabular}{|c|c|c|}
\hline Grupo de sintomas & $\mathbf{N}$ & $\%$ \\
\hline \multicolumn{3}{|l|}{ Humor depressivo/ansioso } \\
\hline Sente-se nervosa, tensa ou preocupada & 1429 & 69,5 \\
\hline Assusta-se com facilidade & 1166 & 56,7 \\
\hline Tem se sentido triste ultimamente & 953 & 46,4 \\
\hline Tem chorado mais do que de costume & 671 & 32,7 \\
\hline \multicolumn{3}{|l|}{ Sintomas somáticos } \\
\hline Tem dores de cabeça freqüentes & 976 & 47,5 \\
\hline Dorme mal & 623 & 30,3 \\
\hline Tem sensações desagradáveis no estômago & 670 & 32,6 \\
\hline Tem má digestão & 514 & 25,0 \\
\hline Tem falta de apetite & 607 & 29,5 \\
\hline Tem tremores nas mãos & 440 & 20,4 \\
\hline \multicolumn{3}{|l|}{ Decréscimo de energia vital } \\
\hline Cansa-se com facilidade & 878 & 42,7 \\
\hline Tem dificuldade em tomar decisões & 896 & 43,6 \\
\hline \multicolumn{3}{|l|}{ Tem dificuldades de realizar com satisfação } \\
\hline suas atividades & 241 & 11,7 \\
\hline Seu trabalho é penoso & 225 & 10,9 \\
\hline Sente-se cansada o tempo todo & 364 & 17,7 \\
\hline Tem dificuldade de pensar com clareza & 650 & 31,5 \\
\hline \multicolumn{3}{|l|}{ Pensamentos depressivos } \\
\hline É incapaz de desempenhar papel útil em sua vida & 141 & 6,9 \\
\hline Tem perdido o interesse pelas coisas & 418 & 20,3 \\
\hline Tem tido a idéia de acabar com a vida & 167 & 8,1 \\
\hline Sente-se uma pessoa inútil, sem préstimo & 234 & 11,4 \\
\hline
\end{tabular}

Transtornos mentais comuns: descrição de fatores associados à sua ocorrência

A prevalência global de transtornos mentais comuns foi muito elevada: $39,4 \%$ (IC95\% variou de $37,3 \%$ a $41,6 \%$ ), atingindo 811 das 2055 mulheres entrevistadas.

Dentre os sintomas avaliados pelo SRQ-20, e agrupados por Iacoponi e Mari, 21 observou-se que o grupo de sintomas predominantes foi o de "Humor depressivo/ansioso" (Tabela 5). Nesse grupo a maioria das questões obteve elevados percentuais. A questão com maior percentual de respostas afirmativas foi "sentir-se nervosa, tensa ou preocupada" $(69,5 \%)$, seguida de "assustar-se com facilidade" $(56,7 \%)$. Sintomas somáticos representaram o segundo grupo de sintomas referidos, destacando-se dores de cabeça frequientes $(47,5 \%)$ e ter sensações desagradáveis no estômago (32,6\%). A presença de sintomas relacionados ao cansaço também foi expressiva. No grupo de "Decréscimo de energia vital", cansar-se com facilidade obteve o maior percentual $(42,7 \%)$, enquanto a referência ao trabalho como penoso apresentou a menor freqüência $(10,9 \%)$. Perda de interesse pelas coisas $(20,3 \%)$ e sentir-se uma pessoa inútil, sem préstimo $(11,4 \%)$ foram os mais referidos no grupo "Pensamentos depressivos".

Analisando-se a ocorrência de transtornos mentais comuns (TMC), segundo variáveis sociodemográficas, observou-se que algumas características estavam estatisticamente associadas à ocorrência de TMC (Tabela 6). A prevalência de TMC foi significantemente mais elevada entre as mulheres que: tinham baixo nível de escolaridade; não moravam 
Prevalência (\%) de transtornos mentais comuns entre mulheres segundo variáveis sociodemográficas.

\begin{tabular}{|c|c|c|c|c|c|c|}
\hline Variáveis sociodemográficas & $\mathbf{N}$ & $\mathrm{n}$ & $\%$ & $P$ & $\mathbf{R P}$ & IC95\% \\
\hline \multicolumn{7}{|l|}{ Idade } \\
\hline Até 40 anos & 1296 & 464 & 35,8 & - & * & - \\
\hline Mais de 40 anos & 759 & 347 & 45,7 & 0,001 & 1,28 & $1,15-1,42$ \\
\hline \multicolumn{7}{|l|}{ Situação conjugal } \\
\hline Solteira & 737 & 233 & 31,6 & - & * & - \\
\hline Casada + União Estável & 954 & 405 & 42,5 & 0,001 & 1,34 & $1,18-1,53$ \\
\hline Viúva/Separada/Desquistada/Divorciada & 356 & 171 & 48,0 & 0,001 & 1,52 & $1,31-1,77$ \\
\hline \multicolumn{7}{|l|}{ Cor da pele (auto-referida) } \\
\hline Parda & 1140 & 459 & 40,3 & 0,023 & 1,26 & $1,08-1,47$ \\
\hline Negra & 352 & 164 & 46,6 & 0,001 & 1,46 & $1,22-1,74$ \\
\hline Branca & 435 & 139 & 32,0 & - & * & - \\
\hline \multicolumn{7}{|l|}{ Escolaridade } \\
\hline Sem acesso à escola & 267 & 152 & 56,9 & 0,001 & 3,05 & $1,90-5,02$ \\
\hline Ensino Fundamental & 898 & 420 & 46,8 & 0,001 & 2,54 & $1,57-4,10$ \\
\hline Ensino médio & 797 & 220 & 27,6 & 0,842 & 1,50 & $0,92-2,44$ \\
\hline Graduação & 76 & 14 & 18,4 & - & * & - \\
\hline \multicolumn{7}{|l|}{ Renda mensal } \\
\hline Até $\mathrm{R} \$ 200,00$ (1 SM) & 476 & 235 & 49,4 & 0,001 & 1,84 & $1,51-2,23$ \\
\hline$>\mathrm{R} \$ 200,00(>1 \mathrm{SM})$ & 346 & 93 & 26,9 & - & * & - \\
\hline \multicolumn{7}{|l|}{ Número de filhos } \\
\hline Sem filhos & 659 & 186 & 28,2 & - & * & - \\
\hline 1 - 2 filhos & 636 & 258 & 40,6 & 0,001 & 1,44 & $1,23-1,68$ \\
\hline 3 - 5 filhos & 393 & 182 & 46,3 & 0,001 & 1,64 & $1,40-1,93$ \\
\hline $6-7$ filhos & 222 & 114 & 51,4 & 0,001 & 2,34 & $2,03-2,70$ \\
\hline$>8$ filhos & 145 & 71 & 49,0 & 0,001 & 1,73 & $1,41-2,13$ \\
\hline
\end{tabular}

* Grupo de referência; $\mathrm{RP}=$ razão de prevalência; IC95\% = intervalo de confiança 95\%; SM = salário mínimo; $\mathrm{N}=$ número total de mulheres em cada estrato; $\mathrm{n}=$ número de mulheres com suspeição de transtorno mental comum.

mais com seus companheiros (eram separadas, divorciadas ou viúvas); eram negras ou pardas; tinham rendimento mensal próprio até um salário mínimo, tinham filhos e eram chefes de família.

Ajuda nas tarefas domésticas, dias da semana que realizava tarefas domésticas e alta sobrecarga doméstica foram características referentes ao trabalho doméstico que estavam estatisticamente associadas aos TMC (Tabela 7). Observou-se que a ajuda doméstica remunerada estava associada à menor prevalência de TMC $(28,0 \%)$, enquanto encontraram-se prevalências elevadas quando a entrevistada não dispunha de nenhum auxílio $(47,1 \%)$ ou contava apenas com a colaboração de um homem $(46,9 \%)$. A prevalência de TMC foi mais elevada entre as mulheres com alta sobrecarga doméstica $(49,5 \%)$ do que entre aquelas com baixa sobrecarga $(33,1 \%)$.

Mulheres que não participavam de atividades regulares de lazer apresentaram maior prevalência de TMC $(49,9 \%)$ do que aquelas que participavam regularmente de algum tipo de atividade de lazer $(31,6 \%)$. Avaliando-se a prevalência de TMC segundo atividade de lazer e número de filhos, observou-se que as mulheres que mantinham atividades de lazer apresentaram prevalências de TMC sempre inferiores às mulheres que não dedicavam tempo ao lazer. Observou-se também que o número de filhos influenciava na prevalência observada: mesmo mantendo atividades de lazer, quando aumentava o número de filhos, aumentava também a prevalência de TMC (Tabela 8). 
Tabela 7

Prevalência (\%) de transtornos mentais comuns entre mulheres segundo características do trabalho doméstico.

\begin{tabular}{|c|c|c|c|c|}
\hline \multirow[t]{2}{*}{ Variáveis sociodemográficas (N) } & \multicolumn{2}{|c|}{$\begin{array}{c}\text { Presença de } \\
\text { transtorno mental }\end{array}$} & \multirow[t]{2}{*}{ RP } & \multirow[t]{2}{*}{$p$} \\
\hline & $\mathrm{n}$ & $\%$ & & \\
\hline \multicolumn{5}{|l|}{ Chefia da família } \\
\hline $\operatorname{Sim}(281)$ & 126 & 44,8 & 1,6 & 0,047 \\
\hline Não (1.774) & 685 & 38,6 & * & * \\
\hline \multicolumn{5}{|l|}{ Ajuda nas tarefas domésticas } \\
\hline Nenhuma (497) & 234 & 47,1 & 1,68 & 0,001 \\
\hline Marido/outro homem (147) & 69 & 46,9 & 1,67 & 0,001 \\
\hline Outra mulher (1.011) & 379 & 37,5 & 1,34 & 0,006 \\
\hline Outra alternativa (59) & 20 & 33,9 & 1,21 & 0,37 \\
\hline Empregada (239) & 67 & 28,0 & * & * \\
\hline \multicolumn{5}{|l|}{ Dias da semana que realiza tarefas domésticas } \\
\hline Todos os dias/segunda a sexta (1.707) & 691 & 40,5 & 1,19 & 0,015 \\
\hline Faz apenas nos finais de semana ou não faz tarefa doméstica (279) & 95 & 34,0 & * & 0,20 \\
\hline \multicolumn{5}{|l|}{ Sobrecarga doméstica } \\
\hline Alta $(550)$ & 272 & 49,5 & 1,50 & 0,001 \\
\hline Média (775) & 298 & 38,5 & 1,16 & 0,029 \\
\hline Baixa (720) & 238 & 33,1 & * & - \\
\hline
\end{tabular}

$\mathrm{RP}=$ razão de prevalência ; * Grupo de referência; $\mathrm{N}=$ número total de mulheres em cada estrato; $\mathrm{n}==$ número de mulheres com suspeição de transtorno mental comum em cada estrato considerado.

\section{Tabela 8}

Prevalência (\%) de transtornos mentais comuns entre mulheres segundo participação regular em atividades de lazer e número de filhos.

\begin{tabular}{|c|c|c|c|c|c|c|}
\hline \multirow[t]{3}{*}{ Numero de filhos $(\mathrm{N}=2054)$} & \multicolumn{3}{|c|}{ Com atividade de lazer } & \multicolumn{3}{|c|}{ Sem atividade de lazer } \\
\hline & \multicolumn{3}{|c|}{ Prevalência } & \multicolumn{3}{|c|}{ Prevalência } \\
\hline & $\mathrm{N}$ & $\mathrm{n}$ & $\%$ & $\mathrm{~N}$ & $\mathrm{n}$ & $\%$ \\
\hline Sem filhos & 480 & 117 & 24,4 & 179 & 69 & 38,5 \\
\hline 1 a 2 filhos & 326 & 113 & 34,7 & 309 & 144 & 46,6 \\
\hline 3 a 4 filhos & 183 & 69 & 37,7 & 210 & 113 & 53,8 \\
\hline 5 a 7 filhos & 79 & 31 & 39,2 & 143 & 83 & 58,0 \\
\hline 8 ou mais filhos & 59 & 26 & 44,1 & 86 & 45 & 52,3 \\
\hline Total & 1127 & 356 & 31,6 & 927 & 454 & 49,0 \\
\hline
\end{tabular}

$\mathrm{N}=$ número total de mulheres em cada estrato; $\mathrm{n}=$ número de mulheres com suspeição de transtorno mental comum em cada estrato considerado.

\section{Discussão}

Estudos de corte transversal produzem instantâneos da situação de saúde, portanto, não podem avaliar adequadamente causalidade entre eventos. ${ }^{8}$ Outro limite importante é o viés de sobrevivência: apenas indivíduos que apresentaram o efeito investigado no momento da pesquisa são analisados. ${ }^{22}$ Esses limites devem ser, portanto, considerados na análise dos resultados obtidos.

Os fatores associados à ocorrência de transtornos mentais comuns na população estudada podem ser 
assim sumarizados: a) ser separada/divorciada ou viúva, ter filhos, ser negra ou parda, ter baixa escolaridade, baixa renda, mais de 40 anos, dentre as características sociodemográficas; b) ter alta sobrecarga doméstica, ser a chefe da família, realizar tarefas domésticas todos os dias da semana e não receber ajuda nas tarefas ou recebê-la apenas de um homem da família, dentre as características do trabalho doméstico; c) não manter, semanalmente, algum tipo de atividade de lazer.

As características sociodemográficas, aqui associadas aos transtornos mentais psíquicos, também têm sido descritas em outros estudos de saúde mental,2,3,5,8 revelando que determinadas características sociodemográficas (tais como baixa escolaridade, cor da pele negra/parda, ausência de um companheiro vivenciada na situação de viuvez ou divórcio e baixo nível de renda) encontram-se fortemente associadas ao adoecimento psíquico.

As questões referentes ao trabalho doméstico têm recebido maior atenção em estudos recentes. 12 Os resultados descritos na literatura ${ }^{8}$ confirmam os resultados deste estudo, especialmente no que se refere ao trabalho doméstico como atribuição ainda exclusivamente feminina e ao não compartilhamento, por parte dos homens, das responsabilidades domésticas.

Os resultados mostram que, mesmo ao dispor de ajuda nas tarefas domésticas, essa permanecia sendo realizada por uma outra mulher e/ou por empregada doméstica; ou seja, a realização do trabalho doméstico compete aos membros femininos da família, observando-se que a proporção de mulheres que não executava tarefas domésticas foi sempre inferior à proporção daquelas que executava. Observa-se, assim, que a forma de organização do trabalho doméstico, ainda hoje, preserva o modelo de família tradicional, mantendo a invisibilidade das atividades realizadas no âmbito privado (o lar) e centralizandoas como atribuição feminina, responsabilidade das mulheres.

Além de constatar o baixo percentual de auxílio masculino na realização das tarefas domésticas, Aquino e Menezes 23 observaram que o apoio masculino, quando existente, reforçava e reproduzia a divisão do trabalho no interior da família. A participação masculina restringia-se a atividades realizadas externamente ao domicílio, no espaço de circulação pública: compras no supermercado, pagamentos em bancos ou, então, quando realizados no espaço doméstico, a pequenos consertos. Tarefas como cozinhar, limpar a casa e, especialmente, o cuidado e a educação dos filhos ficavam a cargo das mulheres. Os autores destacam ainda que as ativi- dades assumidas pelos homens, geralmente realizadas em espaços externos à casa, eram também diferenciadas quanto à dimensão temporal. Ir ao mercado, fazer feira ou realizar pagamentos são atividades que podem ser flexibilizadas, enquanto atividades como cozinhar e cuidar dos filhos são fortemente orientadas ao atendimento em um tempo determinado, em que possíveis arranjos e reordenamentos são bem mais restritos.

A alta sobrecarga doméstica foi expressiva no presente estudo e, confirma, mais uma vez, o predomínio das mulheres na execução das tarefas domésticas básicas (cozinhar, lavar, limpar e passar). Como apontado em outros estudos, 8,23 o cuidado com os filhos também alcançou os maiores percentuais entre as atividades familiares realizadas integralmente ou na maior parte das vezes pelas mulheres. A construção social dos papéis sociais de homens e mulheres tem oferecido sustentáculo a pouca participação masculina nas atividades domésticas. Para Lima,12 a ênfase no papel maternal e doméstico da mulher deixou conseqüências também no que se refere ao papel masculino frente à família, pois permite uma certa "desresponsabilização" do homem quanto ao lar e à vida doméstica, no que não esteja diretamente relacionado ao sustento financeiro da família.

A ausência de apoio na realização das atribuições domésticas, levando à divisão desigual do trabalho, aparece estreitamente ligada à qualidade de vida, especialmente da saúde psíquica. Glass e Fujimoto, 24 por exemplo, observaram que a sobrecarga de trabalho doméstico, assim como do trabalho profissional, aumentava sintomas de depressão. Walters et al.25 também constataram que demandas domésticas tiveram impacto negativo à saúde das mulheres estudadas. Neste estudo, os achados também apontaram que a prevalência de transtornos mentais comuns atingia patamares mais elevados entre aquelas que não recebiam nenhum tipo de auxílio na realização das atribuições domésticas no interior da família.

Achados de outros estudos 7,17 revelam que o tempo dedicado às tarefas domésticas - a duração da jornada de trabalho - varia conforme a estrutura familiar, determinada, principalmente, pelo número de filhos e pelos meios de apoio disponíveis para a realização das tarefas. Na presente pesquisa, observou-se que à medida que aumentava o número de filhos aumentava a prevalência de TMC, e que na maior parte das tarefas domésticas investigadas verificou-se um elevado percentual de mulheres como a maior responsável ou inteiramente responsável pelas tarefas. Portanto, a ausência de apoio e o número 
elevado de filhos, que aumentavam a sobrecarga e a jornada de trabalho, podem interagir para aumentar o sofrimento mental proveniente desse tipo de atividade.

Outro aspecto importante associando saúde mental e trabalho doméstico entre mulheres, referese à distribuição do tempo entre as muitas tarefas a serem executadas cotidianamente. Conflitos com relação à distribuição do tempo são, em geral, mais dramáticos para as mulheres trabalhadoras, embora atinjam todas as mulheres, e toquem em um aspecto muito importante para a saúde mental: o tempo dedicado às atividades prazerosas e, ao cuidado consigo próprio. De acordo com Heller, 26 na organização do tempo, para responder às demandas diárias, as mulheres estabelecem critérios hierárquicos por meio dos quais determinadas tarefas assumem caráter prioritário, enquanto outras, compreendidas como mais flexíveis e menos relevantes são adiadas.

Segundo Carpentier-Roy 27 e Kergoat et al.,28 o processo de construção hierárquica das atividades é fortemente dominado pelo contexto social de produção do imaginário feminino, em que a centralidade do que é rotulado como "boa mulher" apóia-se no desenvolvimento das qualidades necessárias ao cuidado do outro, geralmente edificadas em valores como solidariedade e abnegação. Assim, não é raro a compressão/limitação do tempo que seria dedicado às atividades prazerosas, como as de lazer, em função das necessidades e demandas de outras atividades, voltadas ao cuidado do outro, ao atendimento do que está internalizado como atribuição feminina: o bem-estar da família.

As repercussões negativas à saúde, decorrentes da limitação do tempo para as atividades direcionadas ao distensionamento físico e mental, vêm sendo descritas na literatura. 23 No presente estudo, a ausência de atividades regulares de lazer estava positivamente associada ao aumento da prevalência de TMC, revelando que a impossibilidade ou restrição do tempo livre pode comprometer a saúde psíquica das mulheres, proporcionando sofrimento mental. Nossos resultados sugerem que o aumento da carga de trabalho doméstico, que pode ser indicada aqui pelo aumento do número de filhos, reduz o tempo destinado ao lazer, e podem, por sua vez, associar-se mais fortemente aos efeitos negativos sobre a saúde psíquica das mulheres. Os aspectos relacionados à dimensão de distribuição do tempo entre os vários papéis desempenhados pelas mulheres e as atividades de lazer merecem maior aprofundamento em estudos futuros.

Ao se avaliar trabalho doméstico é necessário referir, ainda que não tenham sido explorados nesse estudo, os aspectos afetivos embricados no dia-a-dia desse tipo de trabalho. Aqui, o produto das atividades é consumido por pessoas diretamente ligadas, afetiva e emocionalmente, a quem realiza as tarefas. A afetividade envolvida na execução das tarefas domésticas é, portanto, elemento relevante a ser considerado, uma vez que pode contribuir para minimizar ou elevar as repercussões negativas sobre a saúde. Nessa perspectiva, deve-se entender que as questões referentes à temática aqui abordadas revestem-se de significativa complexidade, não mantendo relação linear e com direção previamente presumível, pois mescla fatores diversos atuantes em diferentes esferas da vida e da subjetividade feminina. A complexidade das relações que são tecidas justifica e colabora, pelo menos em parte, para a manutenção do modelo de divisão sexual do trabalho no interior das famílias, até o momento atual, centrado nas mulheres.

Por fim, cabe registrar que os achados aqui apresentados são provenientes de análise exploratória; o objetivo foi descrever a prevalência de TMC entre mulheres e explorar fatores relacionados às características sociodemográficas e aspectos do trabalho doméstico, potencialmente associados aos transtornos mentais estudados. Não foi aqui incluída uma análise simultânea dos aspectos investigados, com avaliação de possíveis interações entre as variáveis estudadas. Desse modo, os achados descritos devem ser considerados com cautela. Estudos futuros poderão, a partir dos resultados aqui apresentados, aprofundar a análise iniciada, incluindo métodos multivariados de análise para controle e ajuste das variáveis de interesse.

\section{Conclusões}

Os achados deste estudo foram similares àqueles descritos na literatura.9,12,17,25 Observa-se que a mulher, mesmo após sua inserção no mercado de trabalho, permanece a principal responsável por planejar e executar as atividades domésticas, sem receber ajuda substancial dos companheiros e filhos, sendo o auxílio nas tarefas, quando disponível, proveniente de uma outra mulher.

Nossos resultados sugerem que aspectos relacionados ao trabalho doméstico podem estar associados a repercussões negativas à saúde mental das mulheres. Portanto, o trabalho doméstico não pode mais manter-se invisível, portador de atributos negativos e de pouco valor social.

Além disso, cabe fomentar a discussão sobre a implementação de políticas públicas para criação de 
uma rede de apoio social capaz de disponibilizar meios de socialização de parcela substancial do trabalho doméstico (creches, lavanderias comunitárias, pequenas cooperativas de alimentos prontos). O processo de consolidação de iniciativas de base comunitária voltadas para a constituição

\section{Agradecimentos}

Agradecemos o apoio financeiro recebido do Programa de Apoio à Fixação de Doutores no Estado da Bahia (PRODOC) da Fundação de Apoio à Pesquisa do Estado da Bahia (FAPESB) (Proc. 99104245)

\section{Referências}

1. Goldberg DP, Huxley P. Common mental disorders - A biosocial model. London: Routledge; 1992.

2. Ludermir AB, Melo Filho DA. Condições de vida e estrutura ocupacional associadas a transtornos mentais comuns. Rev Saúde Pública 2002; 36: 213-21.

3. Lopes CS, Faerstein E, Chor D. Eventos produtores de estresse e transtornos mentais comuns: resultados do PróSaúde. Cad Saude Pública 2003; 19: 1713-20.

4. Bertolote JM. A saúde mental da mulher. Rev Med 2001; 8: 25-32.

5. Coutinho ESF. Fatores sociodemográficos e morbidade psiquiátrica menor: homogeneidade e heterogeneidade de efeitos [tese doutorado]. Salvador: Instituto de Saúde Coletiva da Universidade Federal da Bahia; 1995.

6. Araújo TM, Neto AMS, Reis EJFB, Dutra FRD, Azi GR, Alves RL. Trabalho docente e sofrimento psíquico: um estudo entre professores de escolas particulares de Salvador, BA. Rev Faeeba [Fac Edu Est Bahia] 2003; 20: 485-95.

7. Lennon MC. Work conditions as explanations for the relation between socioeconomic status, gender, and psychological disorders. Epidemiol Rev 1995; 17: 120-7.

8. Araújo TM. Distúrbios psíquicos menores entre mulheres trabalhadoras de enfermagem [tese doutorado]. Salvador: Instituto de Saúde Coletiva da Universidade Federal da Bahia; 1999.

9. Lennon MC. Women and mental health: the interaction of job and family conditions. J Health Soc Behav 1992; 35 : 235-47.

10. Ludermir AB. Inserção produtiva, gênero e saúde mental. Cad Saúde Pública 2000; 16: 647-59.

11. Souza-Lobo E. A classe operária tem dois sexos. São Paulo: Brasiliense; 1991.

12. Lima SCC. Trabalho doméstico: uma trajetória silenciosa de mulheres. Rio de Janeiro: Virtual Científica; 2003.

13. OMS (Organização Mundial da Saúde). Relatório sobre a saúde no mundo - saúde mental: nova concepção, nova esperança. Genebra; 2001. dessa rede de apoio tem revelado que essas alternativas podem, por um lado, constituir meios para reduzir a carga de trabalho no interior das famílias, a baixo custo, e, por outro, inaugurar formas de remuneração de atividades até o momento restritas ao âmbito privado.
14. Lwanga SK, Lemeshow R. Sample size determination in health studies a practical manual. Geneva: WHO; 1991.

15. IBGE (Instituto Brasileiro de Geografia e Estatística). Anuário estatístico de Feira de Santana: 2000. Rio de Janeiro; 2001. v. 1.

16. Araújo TM, Cruz MB, Araújo EM, Silva EM, Carvalho DB. Distúrbios psíquicos menores: estimativas de prevalência em população urbana de Feira de Santana - achados de estudo piloto. Rev Saúde Coletiva [Universidade Estadual de Feira de Santana] 2002; 1: 91-7.

17. Aquino EML. Gênero, trabalho e hipertensão arterial: um estudo de trabalhadoras de enfermagem em Salvador, Bahia [tese doutorado]. Salvador: Instituto de Saúde Coletiva da Universidade Federal da Bahia; 1996.

18. Mari JJ. A validity study of a Psychiatric Screening Questionnaire (SRQ-20) in primary care in city of São Paulo. Br J Psychol 1986; 148: 23-6.

19. Borges CH. Transtornos mentais menores entre trabalhadores de uma usina siderúrgica [dissertação mestrado]. São Paulo: Faculdade de Medicina da Universidade de São Paulo; 1990.

20. Palácios M, Jardim S, Ramos A, Silva Filho JF. Validação do Self-Report Questionnaire-20 (SRQ-20) numa população de trabalhadores de um banco estatal no Rio de Janeiro, Brasil. In: Silva Filho JF, Jardim S, organizadores. A danação do trabalho - organização do trabalho e sofrimento psíquico. Rio de Janeiro: Te Cora; 1998. p. 225-41.

21. Iacoponi E, Mari JJ. Reliability and factor structure of the Portuguese version of Self-Reporting Questionnaire. Int J Soc Psychiatry 1988; 3: 213-22.

22. Checkoway H, Pearce N, Kriebel D. Research methods in occupational epidemiology. New York: Oxford University Press; 2004

23. Aquino EML, Menezes GMS. Para pensar no exercício da paternidade: contribuições a partir de um estudo sobre trabalho e saúde de mulheres. In: Silveira P, organizador. Exercício da paternidade. Porto Alegre: Artes Médicas; 1997. p. 131-41. 
24. Glass J, Fujimoto T. Housework, paid work, and depression among husbands and wives. J Health Soc Behav 1994, 35 179-91.

25. Walters V, Lenton R, French S, Eyles J, Mayr J, Newbold B. Paid work, unpaid work and social support: a study of the health of male and female nurses. Soc Sci Med 1996; 43: 1627-36.

26. Heller A. O cotidiano e a história. Rio de Janeiro: Paz e Terra; 1989.
27. Carpentier-Roy MC. Plaisir et souffrance des infirmières au travail, l'empreinte de l'imaginaire féminin. Prevenir 1990; 20: 23-9.

28. Kergoat D, Imbert F, Doare H, Senotier D. Les infirmières et leur coordination - 1988-1989. Paris: Éditions Lamarre; 1992. p. 21-81.

Recebido em 20 de outubro de 2004

Versão final apresentada em 12 de agosto de 2005

Aprovado em 25 de agosto de 2005 